\title{
Multiple giant angiokeratoma of Fordyce on the shaft of the penis masquerading as keratoacanthoma*
}

\author{
Sudip Kumar Ghosh ${ }^{1}$ \\ Megha Agarwal ${ }^{1}$
}

\author{
Shouvik Ghosh ${ }^{1}$
}

DOI: http:/ / dx.doi.org/10.1590/abd1806-4841.20153876

\begin{abstract}
A bstract: The term 'angiokeratoma' includes a wide range of dermatological conditions of hyperkeratotic vascular disorders with a similar histologic combination of hyperkeratosis and superficial dermal vascular ectasia. Angiokeratomas can be classified into localized and systemic forms. Angiokeratoma of Fordyce (AKF) is a localized form of angiokeratoma, clinically characterized by 1- to 6-mm, black, blue, or dark red, dome-shaped papules located on the scrotum, shaft of penis, labia majora, clitoris, inner thigh, and lower abdomen. We describe herein a case of giant angiokeratoma of Fordyce on shaft of the penis in an elderly man, clinically masquerading as keratoacanthoma.
\end{abstract}

Keywords: Angiokeratoma; Keratoacanthoma; Penis

\section{INTRODUCTION}

Angiokeratoma is an umbrella term that covers a range of dermatological conditions of hyperkeratotic vascular disorders with a similar histologic combination of hyperkeratosis and superficial dermal vascular ectasia. Angiokeratomas can be classified into localized and systemic forms. ${ }^{1}$ The localized forms comprise of solitary papular angiokeratoma, which typically occurs on the legs; localized angiokeratoma of the scrotum and vulva (Fordyce type); the congenital form, angiokeratoma circumscriptum naeviforme, which presents as multiple, hyperkeratotic, papular and plaque-like lesions, usually unilaterally on the lower leg, foot, thigh, buttock, and occasionally elsewhere; and bilateral angiokeratoma, which occurs on the dorsa of the fingers and toes (Mibelli type). ${ }^{1,2}$ On the other hand, the generalized form is known as angiokeratoma corporis diffusum (classically found in Fabry's disease).

We report herein a case of angiokeratoma of Fordyce in an elderly man for its unusual morphological presentation.

\section{CASE REPORT}

A 72-year-old man presented with multiple skin eruptions on the shaft of his penis and scrotum for the preceding four years. He had a few episodes of occasional bleeding from these lesions for the same duration. Examination revealed multiple dome-shaped nodules with a smooth shiny surface and a central crateriform ulceration and adherent keratinous plug on the shaft of the penis (Figure 1).

The size of the lesions varied from 1 to $1.5 \mathrm{~cm}$ in diameter. In addition, multiple keratotic papules were also found on the shaft of penis and scrotum. No mucocutaneous lesions were found elsewhere in the body. Palpation of testis and scrotum revealed no other abnormality.

There was no regional lymphadenopathy. Systemic examination was normal. The routine laboratory investigations including complete hemogram and biochemical panels were within normal limits. Ultrasonography of the scrotum was non-contributory. The larger lesions were excised by radiofrequency surgery and sent for histopathological examination

Approved by the Advisory Board and accepted for publication on 28.08.2014

Study conducted at the Department of Dermatology, Venereology, and Leprosy, R.G. Kar Medical College - West Bengal, India.

Financial Support: None.

Conflict of Interest: None.

$1 \quad$ R.G. Kar Medical College - West Bengal, India.

(C)2015 by Anais Brasileiros de Dermatologia 
(Figure 2). Histopathology of the excised specimens revealed numerous dilated, thin-walled vessels in the papillary dermis which were in close proximity to the overlying epidermis (Figure 3). The epidermis showed hyperkeratosis, focal parakeratosis, and presence of keratinous plugs in a few areas. The rete ridges were elongated and surrounded the dilated vascular channels. Based on the clinical and histopathological features, a diagnosis of angiokeratoma of Fordyce was made. The smaller lesions were successfully treated with electrodessication. During one-year follow-up, no recurrence was noted and the patient remained asymptomatic.

\section{DISAUSSION}

Angiokeratoma of Fordyce (AKF) on the scrotum was first described by John Addison Fordyce in

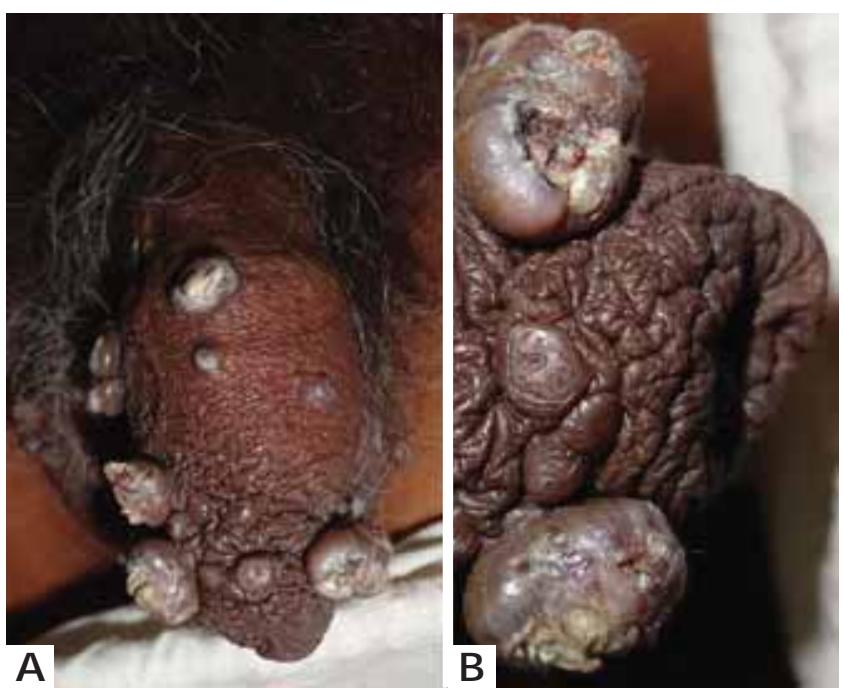

Figure 1: A. Multiple keratotic papules on the shaft of the penis; B. Close-up showing dome-shaped nodules with a smooth shiny surface and a central crateriform ulceration and adherent keratinous plug

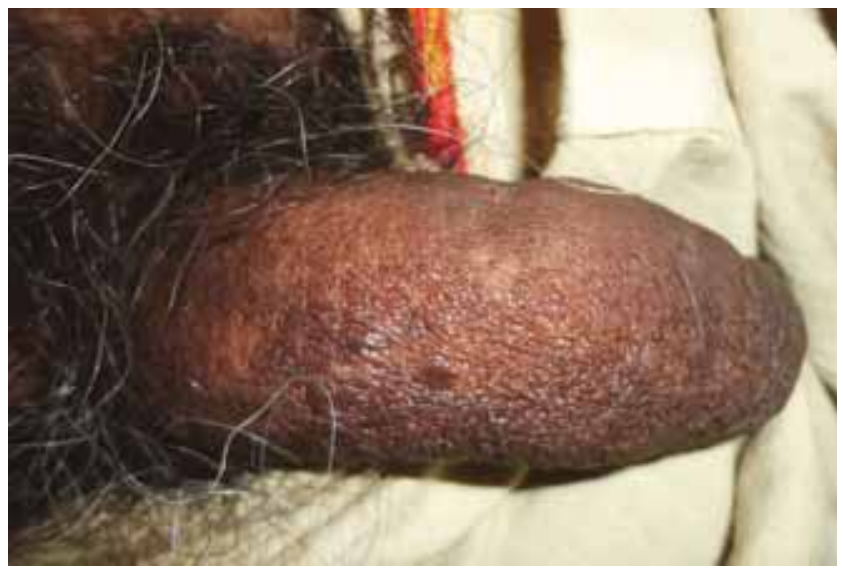

Figure 2: Post-operative view after 4 months of the procedure
1896. These are classically 1- to 6-mm, black, blue, or dark red, dome-shaped papules located on the scrotum, shaft of penis, labia majora, clitoris, inner thigh, and lower abdomen. The lesions become more frequent with increasing age. ${ }^{1,2}$

Giant lesions of angiokeratoma of Fordyce have rarely been described in the literature. The most significant morbidity of this condition comes from bleeding. The papules can bleed spontaneously if traumatized or during intercourse. The lesions are usually bilateral.

However, a few cases of unilateral angiokeratoma of Fordyce have also been described in the literature. ${ }^{3}$ Patients frequently seek medical attention to rule out a sexually transmitted disease or malignancy. The exact pathogenesis of this condition remains elusive. However, increased venous pressure may be a contributory factor to its formation. A number of case reports describe angiokeratomas occurring in the presence of a varicocele or other conditions of increased venous pressure, like hernias, epididymal tumors, urinary system tumors, trauma, thrombophlebitis. Other putative causative factors may include acute or chronic trauma and nevoid or vascular malformations. In a study of vulval angiokeratomas, $54 \%$ of patients were noted to have a predisposing factor (eg, pregnancy, vulval varicosity, post partum, post hysterectomy), while the rest had none. Penile and vulvar angiokeratomas have also been noted after post radiation treatment of genitourinary malignancy. One previous case report has described association of Fabry's disease with angiokeratoma of Fordyce. ${ }^{4}$ The diagnosis of angiokeratoma corporis diffusum should be considered in any patient presenting with scrotal angiokeratomas. ${ }^{1}$ In a recent study, Wang et al. supported the theory of lymphatic derivation of $\mathrm{AKF}$, angiokeratoma corporis diffusum, and solitary angiokeratoma. ${ }^{5}$

Angiokeratoma of Fordyce simulating penile cancer has been described in the literature. ${ }^{6}$ However, the most interesting aspect of the present case was the unusual keratoacanthoma-like clinical presentation, probably a hitherto unreported occurrence. Angiokeratomas of Fordyce have been reported in association with nevus lipomatosus, oral mucosal angiokeratomas, and papular xanthoma. Males have been reported far more often than females, although the exact figures are not available. However, female angiokeratoma cases are probably as common as male cases but are grossly underreported in the literature.

On videodermoscopical examination (x10), AKF is characterized by the presence of dark lacunae, whitish veil, red lacunae, erythema, peripheral erythema, and hemorrhagic crusts.

Dark lacunae, being the most distinctive feature for diagnosis, are sharply demarcated ovoid or round dark blue to black structures corresponding to dilated 

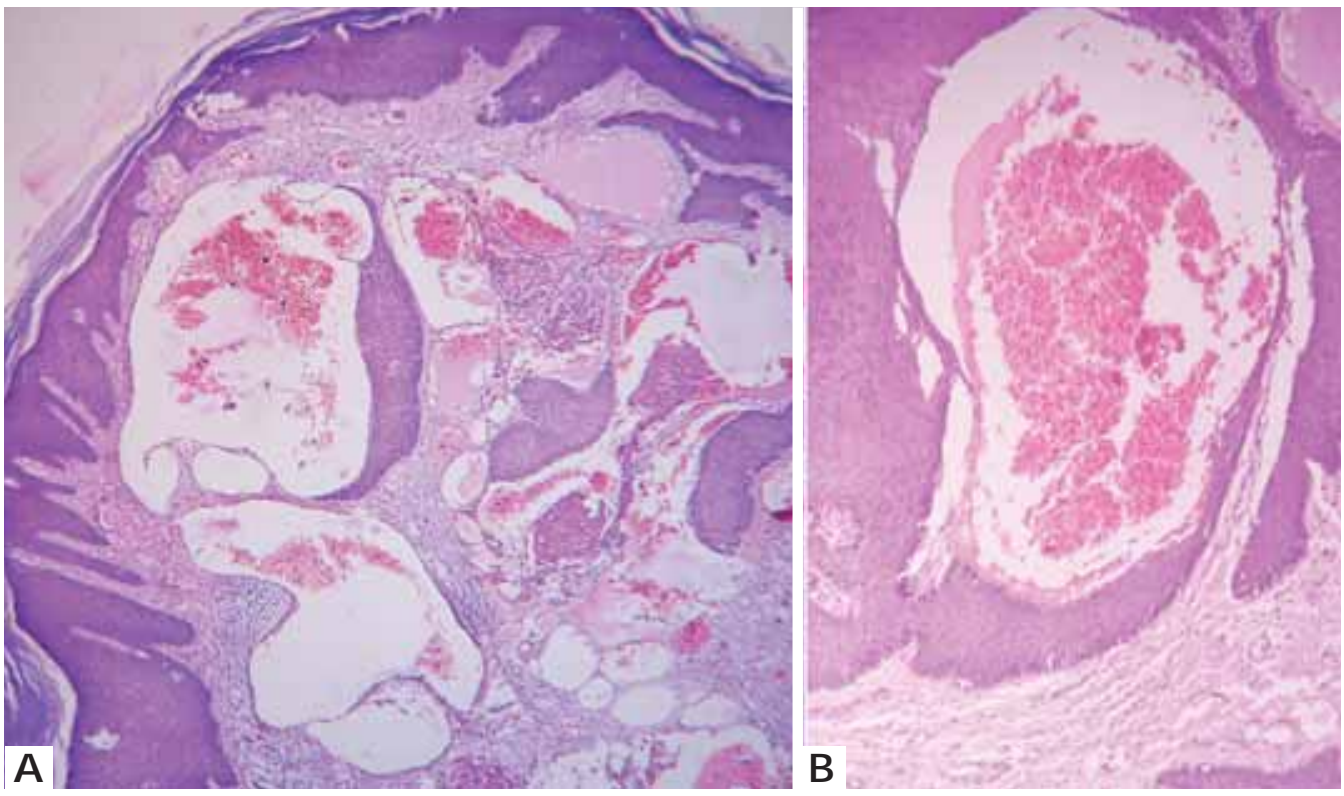

Figure 3: A. The epidermis showing hyperkeratosis, acanthosis, and presence of keratinous plugs in a few areas. Ectatic, thin-walled vessels in the papillary dermis, in close proximity to the overlying epidermis. Some vessels appear to be included within the epidermis. (Hematoxylin-eosin, original magnification $\times 40$ ); B. Dilated vascular channels surrounded by elongated rete ridges (Hematoxylin-eosin, original magnification $\times 100$ ) vascular spaces (which may be partially or completely thrombosed) in the upper dermis. ${ }^{7}$

If the diagnosis is in doubt, then a skin biopsy should be done. ${ }^{2}$ Histopathologically, there is marked dilatation of papillary dermal blood vessels to form large cavernous channels.The overlying epidermis shows hyperkeratosis, irregular acanthosis, and elongation of the rete ridges that partly or completely surround the vascular channels. Additionally, there might be thrombosis of the vessels. ${ }^{2}$
Excision of the lager lesions can be performed, with a good cosmetic result. ${ }^{8}$ Liquid nitrogen cryotherapy and laser ablation may be useful. ${ }^{1,8}$ Light electrocoagulation has been used with or without local anesthesia. ${ }^{1,8} \mathrm{~A}$ previous report of local injections of $0.5 \%$ ethanolamine oleate or $0.25 \%$ sodium tetradecyl sulphate may be a useful procedure with some temporary adverse effects, like mild pain and epithelial sloughing without any scarring. ${ }^{9}$ In a recent report Ichikawa et al demonstrated successful treatment of AKF by SmallSpot Narrow-Band Intense Pulsed Light. ${ }^{10}$

9. Seo SH, Chin HW, Sung HW. Angiokeratoma of Fordyce treated with $0.5 \%$ ethanolamine oleate or $0.25 \%$ sodium tetradecyl sulfate. Dermatol Surg. 2010;36:1634-7.

10. Ichikawa R, Furue M. Successful treatment of scrotal angiokeratomas (Fordyce type) with small-spot narrow-band intense pulsed light. Dermatol Surg. 2013;39:1547-8.

\author{
M AILING ADDRESS: \\ Sudip Kumar Ghosh \\ D epartment of D ermatology, R.G. Kar M edical College \\ 1, Khudiram Bose Sarani \\ Kolkata -700004 \\ West Bengal, India. \\ E-mail: dr_skghosh@yahoo.co.in
}

H ow to cite this article: Ghosh SK, Ghosh S, Agarwal M. Multiple giant angiokeratoma of Fordyce on the shaft of the penis masquerading as keratoacanthoma. An Bras Dermatol. 2015;90 (3 Suppl 1):S150-2. 